\title{
CORRIGENDA
}

\section{Constitutive activation of p38 MAPK in tumor cells contributes to osteolytic bone lesions in multiple myeloma}

J Yang, J He, J Wang, Y Cao, J Ling, J Qian, Y Lu, H Li, Y Zheng, Y Lan, S Hong, J Matthews, MW Starbuck, NM Navone, RZ Orlowski,

$\mathrm{P}$ Lin, LW Kwak and Q Yi

Leukemia (2015) 29, 515; doi:10.1038/leu.2014.265

Correction to: Leukemia (2012) 26, 2114-2123; doi:10.1038/ leu.2012.71

Since the publication of this article, the authors have identified an error contained within Figure 1, namely that it contained the same image for control samples twice, in N2 and N5. In these cases, the histology micrographs were inadvertently duplicated in error when inserting individual image files from a cache of original and cropped sections.
The authors have reviewed the original data from the immunohistochemical staining, and, in each case, identified the correct images that should have been used in Figure $1 \mathrm{~b}$. The correct figure is shown here.

The Authors would like to apologise for any inconvenience this may have caused.

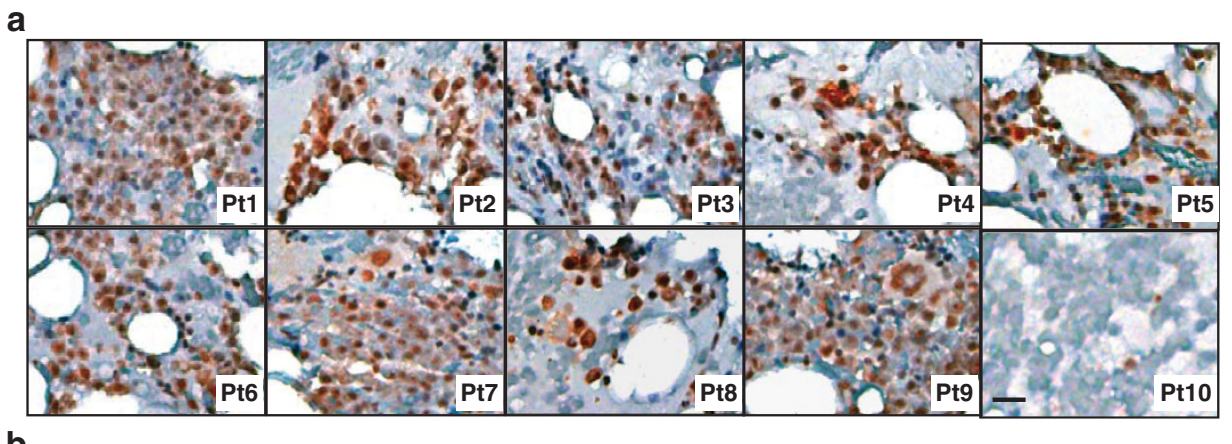

b
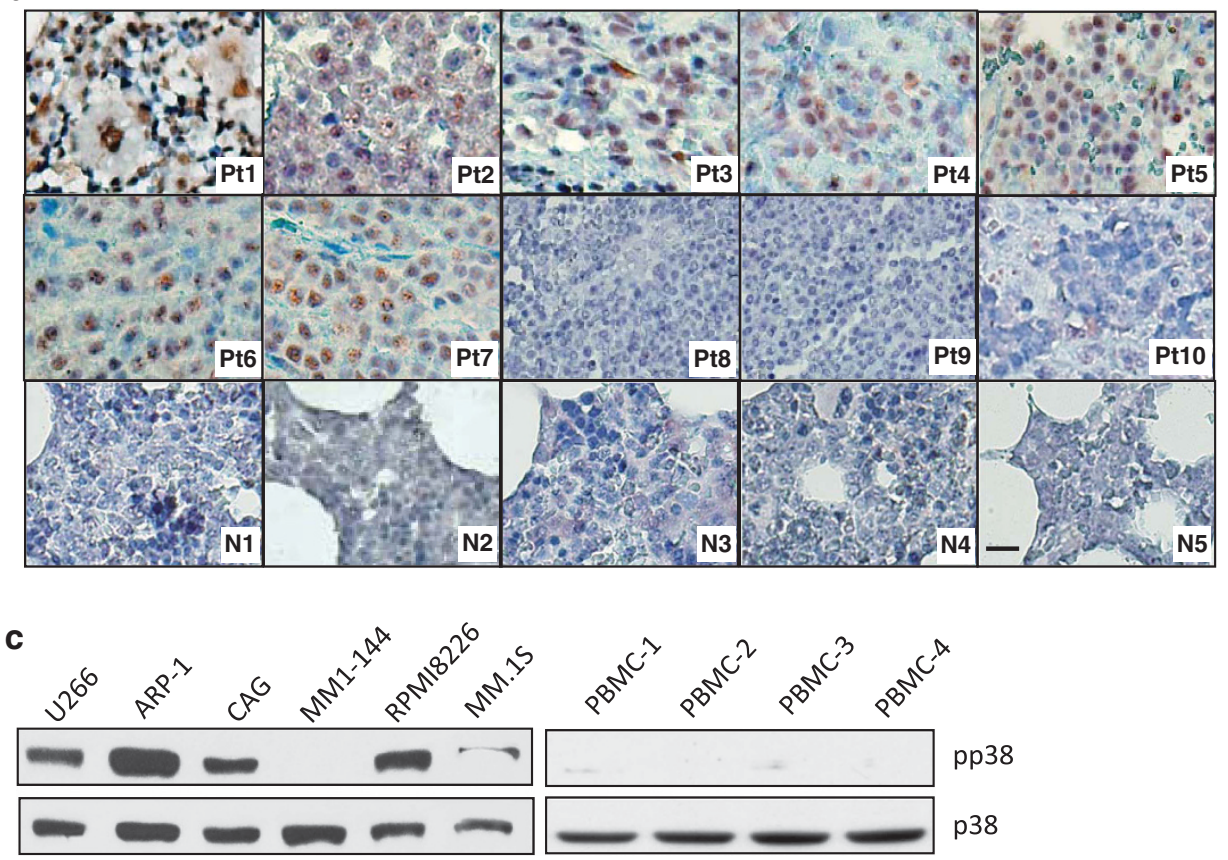

Figure 1. Constitutive activation of p38 in myeloma cells. Representative images of immunohistochemical staining for pp38 in (a) bone marrow biopsy specimens of 10 randomly selected patients with newly diagnosed myeloma (Pt1-Pt10) and (b) a tissue array containing bone marrow samples of 10 myeloma patients (Pt1-Pt10) and 11 healthy donors. (Only five samples, N1-N5; are shown. The other six samples also stained negatively for pp38.) Scale bar, $10 \mathrm{~mm}$. Western blot analysis showing the levels of phosphorylated p38 (pp38) and nonphosphorylated p38 (p38) in (c) six myeloma cell lines and in three normal peripheral blood mononuclear cells. Representative results of three independent experiments are shown. 